\title{
Puerarin inhibits growth and induces apoptosis in SMMC-7721 hepatocellular carcinoma cells
}

\author{
WEI-GUO ZHANG ${ }^{1,2}$, XIAO-FANG LIU ${ }^{2}$, KE-WEI MENG ${ }^{2}$ and SAN-YUAN HU ${ }^{1}$ \\ ${ }^{1}$ Department of General Surgery, Qilu Hospital, Shandong University, Jinan, Shandong 250012; \\ ${ }^{2}$ Department of Hepatobiliary and Pancreatic Surgery, Yantai Yuhuangding Hospital, Yantai, Shandong 264000, P.R. China
}

Received November 13, 2013; Accepted June 6, 2014

DOI: $10.3892 / \mathrm{mmr} .2014 .2512$

\begin{abstract}
Puerarin, a predominant isoflavonoid compound extracted from the Chinese medicinal herb Radix Puerariae, is considered to exhibit an antitumor effect. In the present study, the effects of puerarin on SMMC-7721 human hepatocellular carcinoma cells were investigated. Cell viability was assessed by MTT assay. Apoptosis was detected by flow cytometry with Annexin V-fluorescein isothiocyante staining and morphological observation of nuclear changes by Hoechst staining. The mitochondrial membrane potential (MMP) was monitored using rhodamine 123 . The generation of reactive oxygen species (ROS) was quantified using dichloro-dihydro-fluorescein diacetate. Polymerase chain reaction and western blot analysis were used to detect the expression levels of apoptosis-associated genes. The results revealed that high concentrations of puerarin $(500,1,000$ and $1,500 \mu \mathrm{g} / \mathrm{ml})$ significantly inhibited the proliferation of SMMC-7721 cells in a time- and dose-dependent manner. Simultaneously, apoptotic rates were increased and cell morphology was changed following puerarin treatment. Furthermore, puerarin-induced apoptosis of SMMC-7721 cells was associated with loss of MMP and generation of ROS. Puerarin treatment increased caspase-3,8,9 and apoptosis-inducing factor (AIF) mRNA expression levels in SMMC-7721 cells, while the phosphorylation levels of P38, extracellular signal-regulated kinase (ERK1) and c-Jun N-terminal kinase were also increased. Furthermore, caspase-9 and AIF protein expression was upregulated. In conclusion, puerarin inhibited proliferation and induced apoptosis in SMMC-7721 cells via the mitochondria-dependent pathway; however, the specific mechanisms require further investigation.
\end{abstract}

Correspondence to: Dr San-Yuan Hu, Department of General Surgery, Qilu Hospital, Shandong University, 107 Wenhua Xi Road, Jinan, Shandong 250012, P.R. China

E-mail: hsy250012@163.com

Key words: hepatocellular carcinoma, puerarin, apoptosis, mitochondria

\section{Introduction}

Hepatocellular carcinoma (HCC) is a predominant cause of cancer-associated mortality in numerous countries, particularly in Central and West Africa, and East and Southeast Asia (1). The disease is commonly diagnosed at an advanced stage and recurrence rates are high, typically 30-40\% within five years (2). Patients with advanced HCC have a median survival time of 6-8 months and treatment brings few benefits for these patients (2). Conventional chemotherapeutic and radiotherapeutic treatments are not effective in HCC (3). Recently, patients with advanced HCC have been treated with a comprehensive series of vascular interventional therapeutic agents, but median life expectancies have not been markedly prolonged. Thus, novel therapeutic strategies are required to improve the clinical outcomes for HCC patients. Certain Chinese Traditional Medicines were found to be effective in cancer therapy; drugs including bufalin, berberine and tetrandrine were reported to inhibit the proliferation and induce apoptosis in HCC cells (4-6).

Puerarin, 7-hydroxy-3-(4-hydroxyphenyl)-1-benzopyran4-one 8- $\beta$-D-glucopyranoside $\left(\mathrm{C}_{12} \mathrm{H}_{20} \mathrm{C}_{9}\right)$, is a predominant iso-flavonoid compound extracted from the Chinese medicinal herb Radix Puerariae. This compound has been suggested to be useful in the management of various disorders, including endothelial dysfunction, liver fibrosis, neurotoxicity and bone injury (7-10). Recently, the anticancer properties of puerarin have attracted attention; one study suggested that Pueraria mirifica possesses an estrogenic effect and may inhibit the growth of breast cancer cells at high concentrations, similar to other flavonoids (11). Another two studies reported that pueraria induced apoptosis in HT-29 colon cancer cells, and that a novel puerarin nanosuspension exhibited anticancer activity against colon cancer in vitro and in vivo, with high efficacy and low toxicity $(12,13)$. However, the anticancer effect of puerarin in HCC has, to the best of our knowledge, not been analyzed. Thus, the anticancer effects of puerarin were determined in the SMMC-7721 HCC cell line.

\section{Materials and methods}

Cell line. The SMMC-7721 human HCC cell line was purchased from the the Cell Bank of the Chinese Academy of Sciences (Shanghai, China) and cultured in RPMI 1640 
medium (Gibco, Carlsbad, CA, USA) containing 10\% fetal bovine serum (Hangzhou Sijiqing Biological Engineering Materials Co., Ltd., Hangzhou, China) and ampicillin and streptomycin (Beyotime, Shanghai, China) at $37^{\circ} \mathrm{C}$ in a humidified atmosphere of $95 \%$ air and $5 \% \mathrm{CO}_{2}$.

Cell viability assay. The standard MTT assay was used to assess cell viability. Briefly, cells $\left(5 \times 10^{3}\right.$ cells/well) were seeded in 96-well microtiter plates. Following exposure to various concentrations of puerarin for $48 \mathrm{~h}$ (99\% pure; Sigma-Aldrich, St. Louis, MO, USA), $50 \mathrm{ml}$ MTT solution [2 $\mathrm{mg} / \mathrm{ml}$ in phosphate-buffered saline (PBS); Sigma-Aldrich] was added to each well and the plates were incubated for additional $4 \mathrm{~h}$ at $37^{\circ} \mathrm{C}$. The MTT solution in the medium was aspirated off. To solubilize the formazan crystals formed in viable cells, $200 \mathrm{ml}$ dimethylsulfoxide (DMSO) was added to each well. The absorbance was measured at $490 \mathrm{~nm}$ on a automatic microwell plate reader, using DMSO alone as a blank. All assays were performed in quintuplicate and repeated at least three times.

Cell apoptosis analysis. The cells were seeded at a density of $5 \times 10^{5}$ cells per well in six-well plates, cultured overnight, and then treated with $0,500,1,000$ or $1,500 \mu \mathrm{g} / \mathrm{ml}$ puerarin for either 12 or $24 \mathrm{~h}$. The cells were then harvested and washed with ice-cold PBS. An Annexin V-fluorescein isothiocyanate apoptosis detection kit (KeyGEN Biotech, Nanjing, China) was used to detect cell apoptosis, measured with a FACScan instrument (Becton-Dickinson, Mountain View, CA, USA).

Hoechst staining. The cells were seeded on coverslips on a six-well plate and treated with $0,500,1,000$ or $1,500 \mu \mathrm{g} / \mathrm{ml}$ puerarin for 12 or $24 \mathrm{~h}$. The attached cells were washed with PBS and fixed in freshly prepared $4 \%$ paraformaldehyde for $30 \mathrm{~min}$, then washed with PBS and incubated with $10 \mu \mathrm{g} / \mathrm{ml}$ Hoechst 33258 staining solution (Sigma-Aldrich) for $10 \mathrm{~min}$. Following treatment, the cells were washed with PBS and Antifade Mounting Medium (Beyotime) was added. Apoptosis, indicated by condensed and fragmented nuclei, was observed under a Leica DM 500B fluorescence microscope (Leica Microsystems, Wetzlar, Germany).

Mitochondrial membrane potential (MMP). Rhodamine 123 dye (Rho-123; Sigma-Aldrich) was used to detect the changes in the MMP. Cells $\left(5 \times 10^{4}\right.$ cells/well) were cultured in a 24 -well plate. Following $24 \mathrm{~h}$ exposure to various concentrations of puerarin $(0,500,1,000$ and $1,500 \mu \mathrm{g} / \mathrm{ml})$, the cells were washed with PBS, incubated with $10 \mathrm{mg} / \mathrm{ml}$ Rho-123 and subsequently subjected to flow cytometric analysis using a BD FACScan instrument (Becton Dickinson, Mountain View, CA, USA).

Detection of reactive oxygen species (ROS). Detection of ROS was performed by flow cytometric analysis as described previously (14). In brief, $5 \times 10^{4}$ cells/well were cultured in a 24-well plate. Following $12 \mathrm{~h}$ exposure to puerarin $(0,500$, 1,000 and $1,500 \mu \mathrm{g} / \mathrm{ml}$ ), the cells were washed with PBS and resuspended in complete medium followed by incubation with $0.5 \mu \mathrm{M}$ dihydrorhodamine 123 (Sigma-Aldrich) for $30 \mathrm{~min}$ at $37^{\circ} \mathrm{C}$. ROS fluorescence intensity was determined

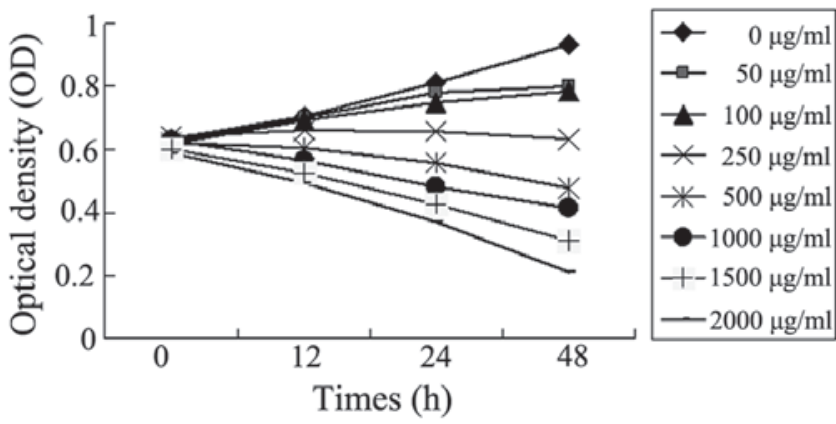

Figure 1. Puerarin inhibits the proliferation of SMMC-7721 human hepatocellular carcinoma cells. The effects of various concentrations of puerarin $(0,50,100,250,500,1,000,1,500$ and $2,000 \mu \mathrm{g} / \mathrm{ml})$ on the viability of SMMC-7721 cells for 12, 24 and $48 \mathrm{~h}$ were assessed by MTT assay. Data are presented as the mean.

by flow cytometry with excitation at $490 \mathrm{~nm}$ and emission at $520 \mathrm{~nm}$.

Quantitative polymerase chain reaction ( $q P C R)$. Cells were seeded at a density of $5 \times 10^{5}$ cells per well in six-well plates, cultured overnight and then treated with $0,30\left(\mathrm{IC}_{25}\right), 500$ $\left(\mathrm{IC}_{50}\right)$ or $2,000 \mu \mathrm{g} / \mathrm{ml}\left(\mathrm{IC}_{75}\right)$ puerarin for $12 \mathrm{~h}$. Total RNA from drug-treated cells was isolated using TRIzol reagent (Invitrogen Life Technologies, Carlsbad, CA, USA). The reverse transcription reaction was conducted using $2 \mu \mathrm{g}$ total RNA with a first strand cDNA kit (Takara Bio, Inc., Shiga, Japan), according to the manufacturer's instructions. PCR amplification was performed for $10 \mathrm{~min}$ at $95^{\circ} \mathrm{C}$, followed by 40 cycles at $95^{\circ} \mathrm{C}$ for $15 \mathrm{sec}$ and annealing/extension at $60^{\circ} \mathrm{C}$ for $45 \mathrm{~s}$ in an ABI 7300 Thermocycler (Applied Biosystems, Foster City, CA, USA), using the SYBR Premix Ex Taq kit (Takara Bio, Inc.). The specific primer sequences for each gene were as follows: Caspase-3, 5'-AACTGGACTGTGGCATTGAG-3' and 5'-ACAAAGCGACTGGATGAACC-3' (product size, 161 bp); caspase-8, 5'-CTGGGAGAAGGAAAGTTG-3' and 5'-TTGGAGAGTCCGAGATTG-3' (product size, 184 bp); caspase-9, 5'-GGAAGAGGGACAGATGAATG-3' and 5'-TTGTTTGGCACCACTCAG-3' (product size, $242 \mathrm{bp}$ ); apoptosis-inducing factor (AIF), 5' - GCTACA A G CACGCTCTA ACA TC-3' and 5'-CAGCCAATCTTCCACTCACAAC-3' (product size, 119 bp); GAPDH, 5'-CACCCACTCCTCCACCTTTG-3' and 5'-CCACCACCCTGTTGCTGTAG-3' (product size, 110 bp). Data analysis was conducted using the $2^{-\Delta \Delta C T}$ method for relative quantification and all sample expression levels were normalized to those of GAPDH, which served as an endogenous control.

Western blot analysis. Cells were seeded at a density of $5 \times 10^{5}$ cells per well in six-well plates, cultured overnight and then treated with $500 \mu \mathrm{g} / \mathrm{ml}$ puerarin for 1,3 and $6 \mathrm{~h}$. Cell lysates were made with standard methods, then $20 \mu \mathrm{g}$ protein samples were separated by $10 \%$ SDS-PAGE, and transferred to polyvinylidene fluoride membranes (PVDF; Roche Diagnostics, Manheim, Germany). After blocking with a buffer containing 5\% low fat milk and $0.1 \%$ Tween-20 in Tris-buffered saline (TBST), the membrane was incubated with mouse monoclonal anti-human primary antibodies 
A

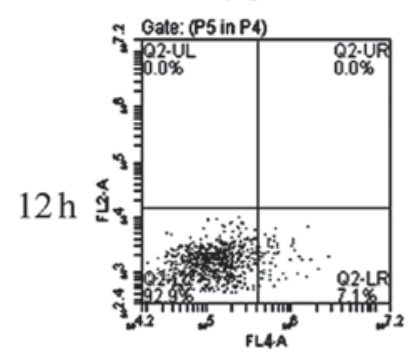

$500 \mu \mathrm{g} / \mathrm{ml}$
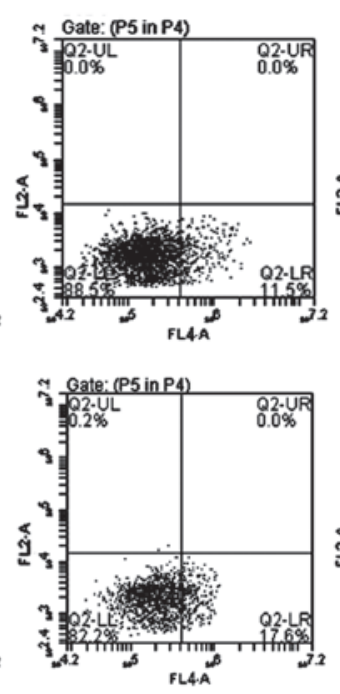

$1000 \mu \mathrm{g} / \mathrm{ml}$
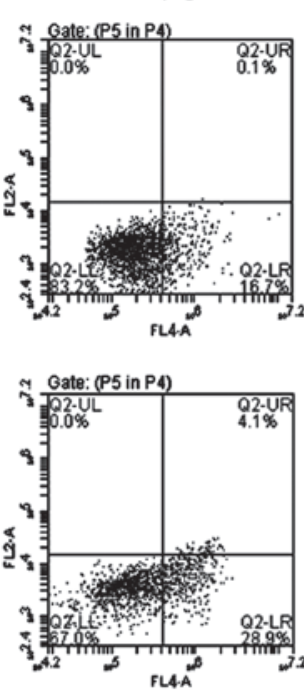

$1500 \mu \mathrm{g} / \mathrm{ml}$
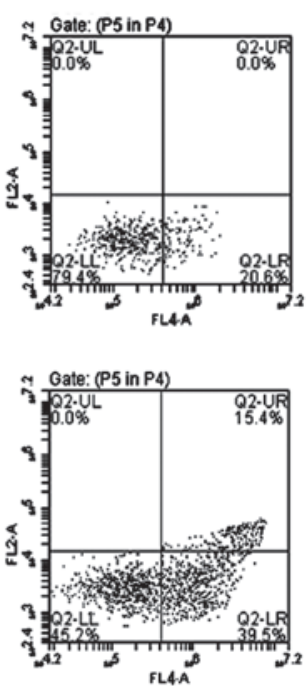

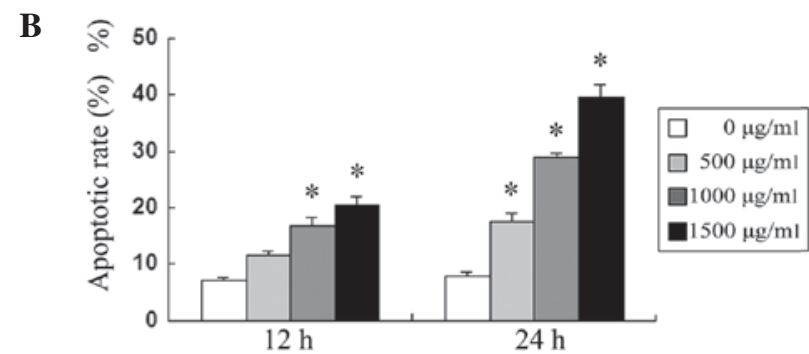

Figure 2. Flow cytometric analysis of apoptosis in SMMC-7721 human hepatocarcinoma cells treated with puerarin. Cells were incubated for 12 and $24 \mathrm{~h}$ with $0,500,1,000$ and 1,500 $\mu \mathrm{g} / \mathrm{ml}$ puerarin. (A) Representative results. (B) Data pooled from three independent experiments reveal the percentage of apoptotic cells. " $\mathrm{P}<0.05$ as compared with the untreated cells.

against AKT1, phosphorylated (p-)AKT, P38, p-P38, extracellular signal-regulated kinase 1 (ERK1), p-RK1, c-Jun N-terminal kinase (JNK), p-JNK, AIF and caspase-3,8 and 9 (Univ-Bio Inc., Shanghai, China) and then incubated with secondary bovine anti-mouse IgG antibody (Santa Cruz Biotechnology, Inc., Santa Cruz, CA, USA). Finally, results were photographed with an enhanced chemiluminescence substrate (horseradish peroxidase, cat no. WBKLS0100; Millipore, Billerica, MA, USA). Protein loading was estimated using mouse anti-GAPDH monoclonal antibody. Lab Works Image Acquisition and Analysis Software version 4.5 (UVP, Upland, CA, USA) was used to quantify band intensities.

Statistical analysis. The SPSS 16.0 software system (SPSS, Inc., Chicago, IL, USA) was used for statistical analysis. Data are expressed as the mean \pm standard error. The differences between groups were analyzed using Student's t-test for two group comparisons or one-way analysis of variance when more than two groups were compared. All tests performed were two-sided. $\mathrm{P}<0.05$ was considered to indicate a statistically significant difference.

\section{Results}

SMMC-7721 cell growth following puerarin treatment. The effects of 12,24 and $48 \mathrm{~h}$ puerarin treatment on the viability of
SMMC-7721 cells were assessed by MTT assay. As shown in Fig. 1, high concentrations of puerarin $(500,1,000,1,500$ and $2,000 \mu \mathrm{g} / \mathrm{ml}$ ) inhibited the proliferation of SMMC-7721 cells.

Apoptotic rate of SMMC-7721 cells treated with puerarin. The treatment of SMMC-7721 cells with puerarin resulted in a significant increase in apoptotic rates in a time- and dose-dependent manner, quantified by Annexin $\mathrm{V}$ analysis (Fig. 2). In concordance with the cell viability rate results, depicted in Fig. 1, these results suggested that the induction of apoptosis may be the main mechanism of the antiproliferative effect of puerarin in SMMC-7721 cells.

Morphological changes of SMMC-7721 cells treated with puerarin. To verify puerarin-induced apoptosis, the morphological changes in SMMC-7721 cells were observed using Hoechst 33258 staining (Fig. 3). Following puerarin treatment, the blue emission became markedly brighter than that in the control cells, indicating a higher rate of apoptosis. Condensed chromatin was also identified in a number of puerarin-treated cells and apoptotic body-like structures were formed in the treated cells.

Induction of apoptosis via the mitochondrial pathway following puerarin treatment. The loss of MMP is associated with activation of the mitochondrial apoptotic pathway. To assess the effect of puerarin on the changes of MMP in 
$0 \mu \mathrm{g} / \mathrm{ml}$
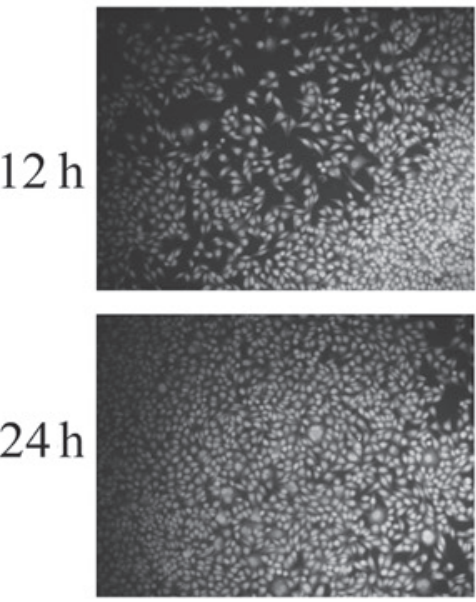

$500 \mu \mathrm{g} / \mathrm{ml}$
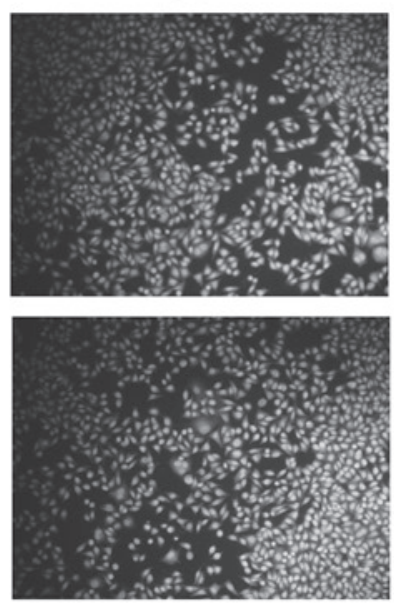

$1000 \mu \mathrm{g} / \mathrm{ml}$
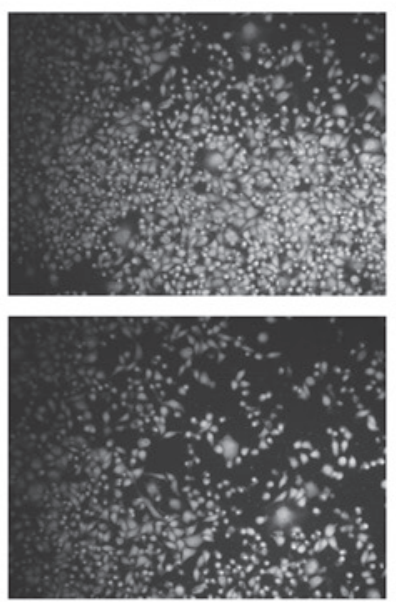

$1500 \mu \mathrm{g} / \mathrm{ml}$
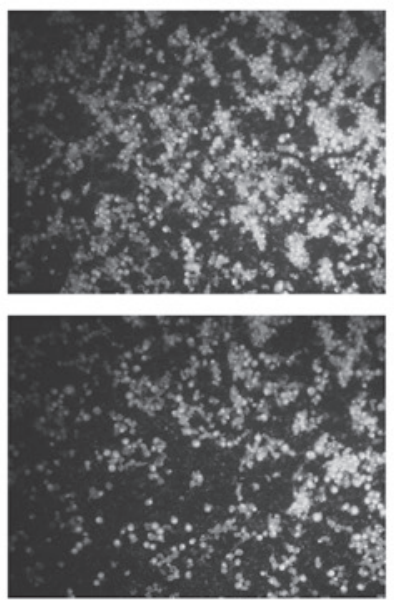

Figure 3. Morphological changes of SMMC-7721 human hepatocarcinoma cells exposed to puerarin. Cells were incubated for 12 and $24 \mathrm{~h}$ with $0,500,1,000$ and $1,500 \mu \mathrm{g} / \mathrm{ml}$ puerarin. The cells were then fixed and stained with Hoechst 33258 and observed under a fluorescent microscope (magnification, x200). Puerarin treatment significantly induced the quantity of condensed chromatin and fragmented nuclei.

A

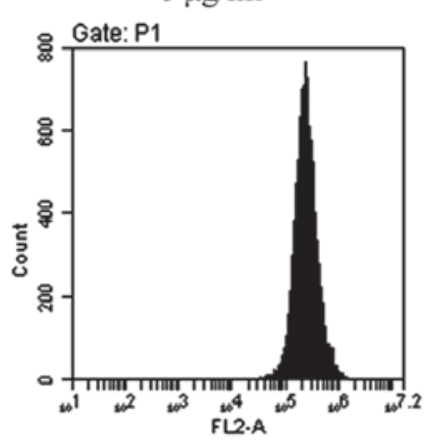

$500 \mu \mathrm{g} / \mathrm{ml}$

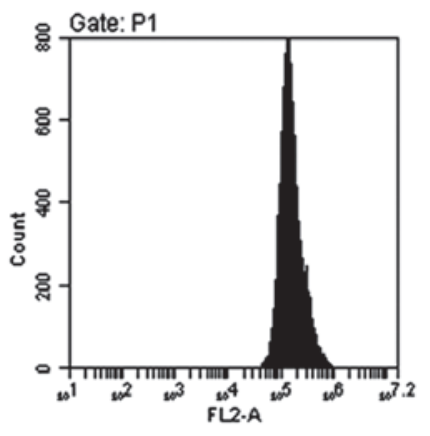

B

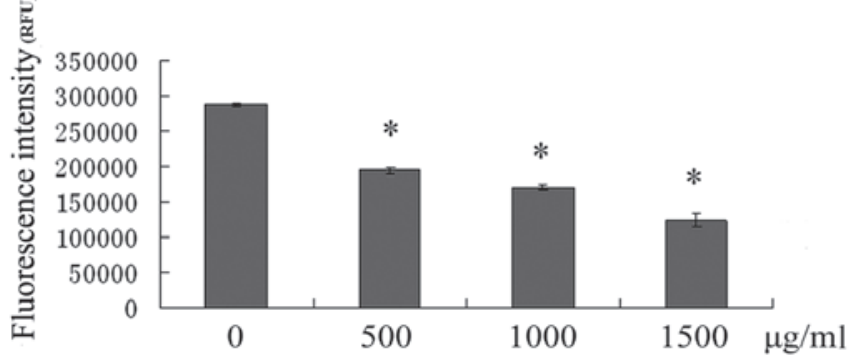

$1000 \mu \mathrm{g} / \mathrm{ml}$
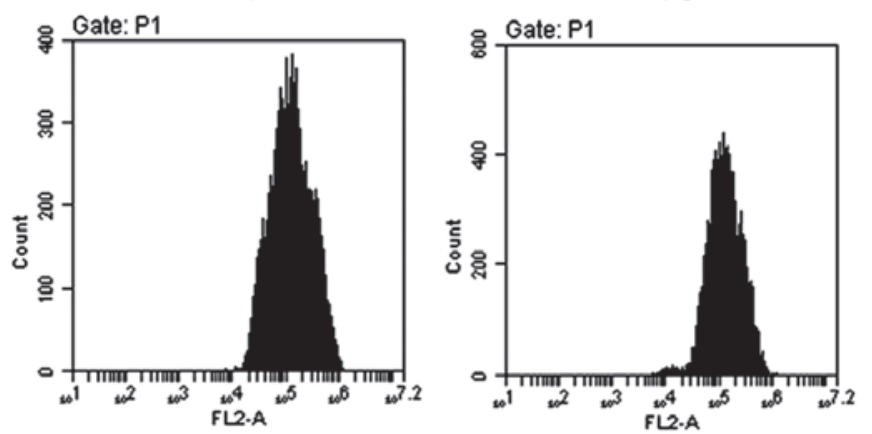

Figure 4. Puerarin reduces the mitochondrial membrane potential in SMMC-7721 human hepatocarcinoma cells. The cells were treated with 0, 500, 1,000 and $1,500 \mu \mathrm{g} / \mathrm{ml}$ puerarin for $24 \mathrm{~h}$, then incubated with rhodamine 123 and analyzed by flow cytometry. (A) Representative results. (B) Data pooled from three independent experiments analyzing fluorescence intensity. ${ }^{*} \mathrm{P}<0.05$ as compared with the untreated cells.

SMMC-7721 cells, flow cytometric analysis was performed to detect the fluorescence intensity of Rho-123. As shown in Fig. 4, treatment of SMMC-7721 cells with 500, 1,000 and $1,500 \mu \mathrm{g} / \mathrm{ml}$ puerarin for $24 \mathrm{~h}$ resulted in a significant depolarization of MMP in a dose-dependent manner $(\mathrm{P}<0.05)$.

ROS generation is also associated with mitochondria. The dichloro-dihydro-fluorescein diacetate fluorescence probe was used to determine the levels of ROS production in SMMC-7721 cells. As shown in Fig. 5, cells exposed to 500, 1,000 and $1,500 \mu \mathrm{g} / \mathrm{ml}$ puerarin for $12 \mathrm{~h}$ exhibited a significant increase in the intracellular accumulation of ROS in a dose-dependent manner $(\mathrm{P}<0.05)$.
Expression levels of apoptosis-associated genes. To clarify the mechanism of SMMC-7721 cell apoptosis induced by puerarin, the mRNA expression levels of apoptosis-associated molecules were determined by qPCR. As shown in Fig. 6A, the expression levels of caspase-3,8,9 and AIF mRNA, particularly those of caspase- 9 , were increased in a dose-dependent manner following puerarin treatment for $12 \mathrm{~h}$.

Subsequently, western blot analysis was used to detect the expression levels of apoptosis-associated proteins and the phosphorylation levels of kinases. As shown in Fig. 6B, treatment with $500 \mu \mathrm{g} / \mathrm{ml}$ puerarin for 3 or $6 \mathrm{~h}$ increased AKT, P38, ERK1 and JNK phosphorylation in a time-depen- 
A

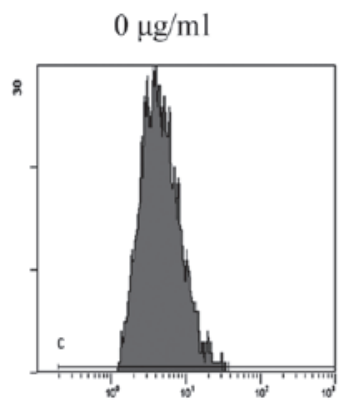

$500 \mu \mathrm{g} / \mathrm{ml}$

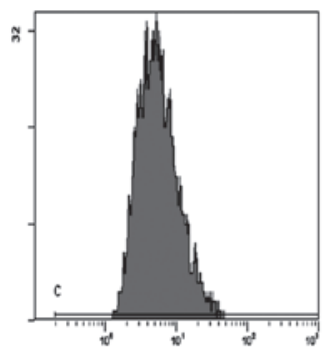

$1000 \mu \mathrm{g} / \mathrm{ml}$

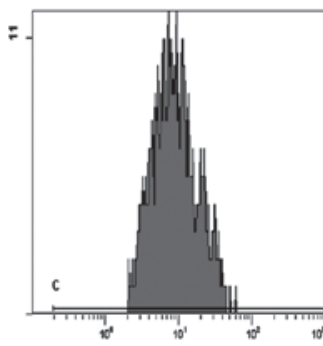

$1500 \mu \mathrm{g} / \mathrm{ml}$

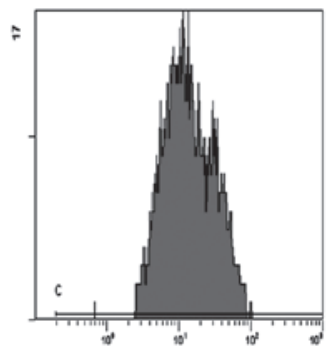

B

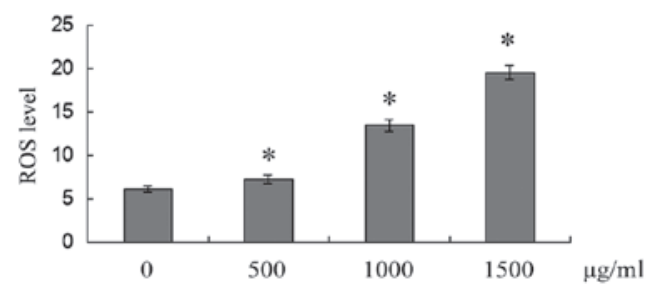

Figure 5. Puerarin increases the number of intracellular ROS in SMMC-7721 human hepatocellular carcinoma cells. The cells were treated with $0,500,1,000$ and $1,500 \mu \mathrm{g} / \mathrm{ml}$ puerarin for $24 \mathrm{~h}$. A dichloro-dihydro-fluorescein diacetate fluorescence probe was used to determine the levels of ROS production; (A) Representative results. (B) Data pooled from three independent experiments indicate the ROS levels. ${ }^{*} \mathrm{P}<0.05$ as compared with the untreated cells; ROS, reactive oxygen species.

dent manner. caspase-9 and AIF protein expression was upregulated.

\section{Discussion}

Radix Puerariae, the root of Pueraria lobata, is extensively used in Chinese Medicine and may also serve as a food in oriental countries. Radix Puerariae contain a large quantity of isoflavones, including puerarin, daidzein, daidzin and genistein. These flavonoids possess various beneficial biological properties, including antiallergic, anti-inflammatory, antiviral, antioxidant and antitumor activities (15). One isoflavone, puerarin, has been shown to exhibit beneficial effects in liver diseases, including cirrhosis and alcohol-induced liver injury $(8,16)$. Furthermore, numerous studies have demonstrated the anticancer activity of puerarin in animal models, as well as proliferation inhibition and apoptosis induction in a variety of cancer cell lines in vitro. One study found that puerarin treatment resulted in a dose-dependent inhibition of cell growth in HS578T, MDA-MB-231 and MCF-7 breast cancer cell lines (11). Results from cell cycle distribution and apoptosis assay studies revealed that puerarin induced cell apoptosis through a caspase-3-dependent pathway and mediated cell cycle arrest in the G2/M phase (11). Similarly, c-jun was found to be downregulated in RL95-2 endometrial carcinoma cells following low-dose puerarin treatment, resulting in reduced aromatase P450 expression levels and substantially decreased growth of endometrial cancers with P450 overexpression (17). In addition, puerarin reduced cell proliferation in acute myeloid leukemia and promyelocytic leukemia cells through cell cycle arrest in $\mathrm{S}$ phase or induction of apoptosis $(18,19)$. Recently, newly developed puerarin nanosuspensions exhibited an enhanced antiproliferative effect by reducing cell viability and inducing morphological changes. The puerarin nanosuspensions inhibited HT-29 human colon cancer cell growth by the induction of early apoptosis. In vivo, the puerarin nanosuspensions were better tolerated and induced
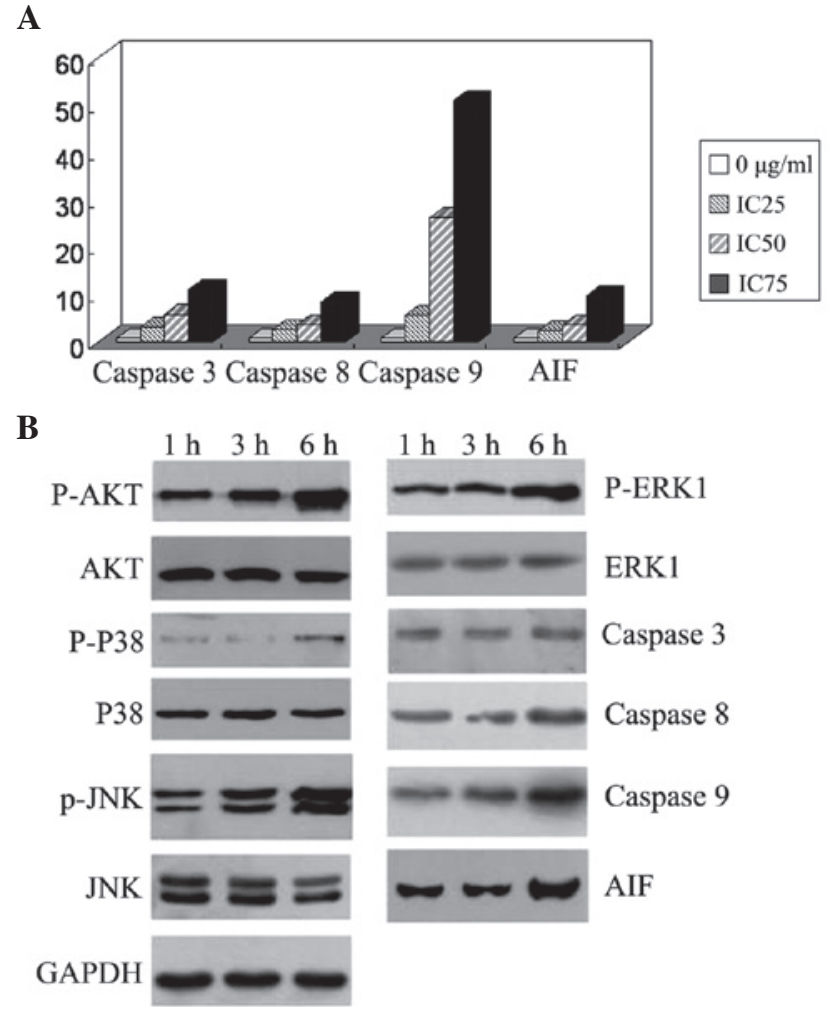

Figure 6. Expression levels of apoptosis-associated genes in SMMC-7721 human hepatocellular carcinoma cells. (A) Cells were incubated for $12 \mathrm{~h}$ with $0,30\left(\mathrm{IC}_{25}\right), 500\left(\mathrm{IC}_{50}\right)$ and $2,000 \mu \mathrm{g} / \mathrm{ml}\left(\mathrm{IC}_{75}\right)$ puerarin. Reverse transcription polymerase chain reaction revealed that the mRNA expression levels of caspase-3,8,9 and AIF, particularly caspase-9, were increased in a dose-dependent manner. (B) Cells were treated with $500 \mu \mathrm{g} / \mathrm{ml}$ puerarin for 1, 3 and $6 \mathrm{~h}$. The AKT, P38, and JNK phosphorylation levels were increased, and caspase-9 and AIF protein expression levels were upregulated. AIF, apoptosis-inducing factor; $\mathrm{p}$-ERK1, phosphorylated extracellular signal-regulated kinase 1; JNK, c-Jun N-terminal kinase.

significantly higher anticancer efficacy than the puerarin-free solution (13). Furthermore, the inhibitory effects of puerarin 
on the invasive and metastatic abilities of tumor cells have also been demonstrated $(20,21)$.

In accordance with previous reports, the present study confirmed that high concentrations of puerarin significantly inhibited proliferation of SMMC-7721 cells in a time- and dose-dependent manner. Simultaneously, apoptotic rates were increased and cell morphology was changed following puerarin treatment, which suggested that induction of apoptosis may be the main mechanism of the antiproliferative effect of puerarin in SMMC-7721 cells.

Apoptosis, programed cell death, is the predominant mechanism for targeted chemotherapies that induce cancer cell death or sensitize the cells to established cytotoxic drugs or radiation treatment. Two signaling pathways that result in apoptosis have been identified: The extrinsic cell death pathway (cell death receptor pathway) and the intrinsic cell death pathway (the mitochondria-initiated pathway) (22). The breakdown of the MMP occurs at an early stage of the apoptotic process and precedes nuclear disintegration disruption. During MMP breakdown, mitochondrial membrane pores are opened, resulting in the loss of MMP (23). This results in an increase in the permeability of the mitochondrial membrane, followed by the release of proapoptotic molecules, such as cytochrome $\mathrm{c}$, released from the mitochondria. Cytochrome $\mathrm{c}$ interacts with adenosine triphosphate, apoptotic protease activating factor 1 and caspase-9, and subsequently activates caspase-3, which consequently elicits caspase-dependent apoptotic cell death (24). In the present study, caspase-3, -8 and -9 as well as AIF mRNA expression levels were significantly increased following puerarin treatment for $12 \mathrm{~h}$, while caspase-9 and AIF protein expression was also upregulated following puerarin treatment for $6 \mathrm{~h}$.

ROS production and consequent oxidative stress have long been implicated in cell apoptosis (25). A previous study found that ROS are predominantly generated in the mitochondria (26). Theoretically, as a consequence of excessive ROS generation in cells, mitochondrial dysfunction may occur (27). As expected, in the present study, the intracellular ROS were significantly increased when SMMC-7721 cells were exposed to puerarin. Therefore, the mitochondria-dependent pathway is important in puerarin-induced apoptosis in SMMC-7721 cells.

Accumulating evidence indicated that activation of mitogen-activated protein kinases (MAPKs) is associated with cell cycle arrest and the induction of apoptosis $(28,29)$. Three predominant parallel MAPK family signaling pathways have been identified: ERK, JNK and P38. Activation of the ERK signaling pathway by puerarin in human osteoblasts has been reported (30). In the present study, the phosphorylation levels of ERK, JNK and P38 in SMMC-7721 cells were all increased following puerarin treatment. AKT is a master regulator involved in transcriptional regulation of the antiapoptotic protein B-cell lymphoma 2, which is critical in the prevention of cell death. Activation of the phosphoinositide 3-kinase/AKT signaling pathway was observed to be involved in the protective effect of puerarin against iodide-induced SH-SY5Y neuroblastoma cell death (31). In the present study, the AKT phosphorylation levels were increased, which may be an adaptive response. Furthermore, in a human breast cancer multidrug-resistant cell line, nuclear factor kappa-B activity and IkappaB degradation were inhibited by puerarin (32).
Puerarin stimulated AMP-activated protein kinase, acetyl-CoA carboxylase and glycogen synthase kinase-3beta phosphorylation, but reduced cAMP-responsive element-binding protein phosphorylation (32). Thus, the anticancer mechanisms of puerarin may be multi-target and therefore require further analysis.

In conclusion, in the present study, puerarin inhibited proliferation and induced apoptosis in SMMC-7721 cells via the mitochondria-dependent pathway, which may provide a novel, safe and effective option for the treatment of HCC in the future.

\section{References}

1. Llovet JM, Burroughs A and Bruix J: Hepatocellular carcinoma. Lancet 362: 1907-1917, 2013.

2. Li C, Wen TF, Liao ZX, et al: Recurrence of hepatocellular carcinoma after liver transplantation: recurrence characteristics and risk factors. Hepatogastroenterology 57: 567-570, 2010

3. Gu W, Fang FF, Li B, Cheng BB and Ling CQ: Characterization and resistance mechanisms of a 5-fluorouracil-resistant hepatocellular carcinoma cell line. Asian Pac J Cancer Prev 13: 4807-4814, 2012

4. Hou Q, Tang X, Liu H, et al: Berberine induces cell death in human hepatoma cells in vitro by downregulating CD147. Cancer Sci 102: 1287-1292, 2011.

5. Liu C, Gong K, Mao X and Li W: Tetrandrine induces apoptosis by activating reactive oxygen species and repressing Akt activity in human hepatocellular carcinoma. Int J Cancer 129: 1519-1531, 2011.

6. Qiu DZ, Zhang ZJ, Wu WZ and Yang YK: Bufalin, a component in Chansu, inhibits proliferation and invasion of hepatocellular carcinoma cells. BMC Complement Altern Med 13: 185, 2013.

7. Huang F, Liu K, Du H, Kou J and Liu B: Puerarin attenuates endothelial insulin resistance through inhibition of inflammatory response in an IKK $\beta /$ IRS-1-dependent manner. Biochimie 94: 1143-1150, 2012.

8. Li R, Liang T, He Q, et al: Puerarin, isolated from Kudzu root (Willd.), attenuates hepatocellular cytotoxicity and regulates the GSK-3 $3 / \mathrm{NF}-\kappa \mathrm{B}$ pathway for exerting the hepatoprotection against chronic alcohol-induced liver injury in rats. Int Immunopharmacol 17: 71-78, 2013.

9. Li R, Liang T, Xu L, et al: Puerarin attenuates neuronal degeneration in the substantia nigra of 6-OHDA-lesioned rats through regulating BDNF expression and activating the Nrf2/ARE signaling pathway. Brain Res 1523: 1-9, 2013.

10. Zhang MY, Qiang H, Yang HQ, Dang XQ and Wang KZ: In vitro and in vivo effects of puerarin on promotion of osteoblast bone formation. Chin J Integr Med 18: 276-282, 2012.

11. Lin YJ, Hou YC, Lin CH, et al: Puerariae radix isoflavones and their metabolites inhibit growth and induce apoptosis in breast cancer cells. Biochem Biophys Res Commun 378: 683-688, 2009.

12. $\mathrm{Yu} \mathrm{Z}$ and $\mathrm{Li} \mathrm{W}$ : Induction of apoptosis by puerarin in colon cancer HT-29 cells. Cancer Lett 238: 53-60, 2006.

13. Wang Y, Ma Y, Zheng Y, et al: In vitro and in vivo anticancer activity of a novel puerarin nanosuspension against colon cancer, with high efficacy and low toxicity. Int J Pharm 441: 728-735, 2013.

14. Jiang CP, Ding H, Shi DH, et al: Pro-apoptotic effects of tectorigenin on human hepatocellular carcinoma HepG2 cells. World J Gastroenterol 18: 1753-1764, 2012.

15. Zhang Z, Lam TN and Zuo Z: Radix Puerariae: an overview of its chemistry, pharmacology, pharmacokinetics, and clinical use. J Clin Pharmacol 53: 787-811, 2013.

16. Guo C, Xu L, He Q, et al: Anti-fibrotic effects of puerarin on CCl4-induced hepatic fibrosis in rats possibly through the regulation of PPAR- $\gamma$ expression and inhibition of PI3K/Akt pathway. Food Chem Toxicol 56: 436-442, 2013.

17. Yu C, Li Y, Chen H, Yang S and Xie G: Decreased expression of aromatase in the Ishikawa and RL95-2 cells by the isoflavone, puerarin, is associated with inhibition of c-jun expression and AP-1 activity. Food Chem Toxicol 46: 3671-3676, 2008.

18. Shao HM, Tang YH, Jiang PJ, et al: Inhibitory effect of flavonoids of puerarin on proliferation of different human acute myeloid leukemia cell lines in vitro. Zhongguo Shi Yan Xue Ye Xue Za Zhi 18: 296-299, 2010 (In Chinese). 
19. Ji O, Shen Q and Si YJ: Effect of flavonoids of puerarin on the proliferation and apoptosis of retinoic acid resistant acute promyelocytic leukemia cell line NB4-R1 cells. Zhonghua Xue Ye Xue Za Zhi 34: 455-457, 2013 (In Chinese).

20. Wang D, Liu Y, Han J, et al: Puerarin suppresses invasion and vascularization of endometriosis tissue stimulated by 17ß-estradiol. PLoS One 6: e25011, 2011.

21. Han J, Yu CQ and Shen W: Inhibitory effects of puerarin on invasion and metastasis of oophoroma cells HO-8910. Zhongguo Zhong Xi Yi Jie He Za Zhi 29: 632-635, 2009 (In Chinese).

22. Xiong Y, Lu QJ, Zhao J and Wu GY: Metformin inhibits growth of hepatocellular carcinoma cells by inducing apoptosis via mitochondrion-mediated pathway. Asian Pac J Cancer Prev 13: 3275-3279, 2012.

23. Zamzami N, Marchetti P, Castedo M, et al: Inhibitors of permeability transition interfere with the disruption of the mitochondrial transmembrane potential during apoptosis. FEBS Lett 384: 53-57, 1996.

24. Yan SL, Huang CY, Wu ST and Yin MC: Oleanolic acid and ursolic acid induce apoptosis in four human liver cancer cell lines. Toxicol In Vitro 24: 842-848, 2010.

25. Yang L, Wang $\mathrm{P}$, Wang $\mathrm{H}$, et al: Fucoidan derived from Undaria pinnatifida induces apoptosis in human hepatocellular carcinoma SMMC-7721 cells via the ROS-mediated mitochondrial pathway. Mar Drugs 11: 1961-1976, 2013.

26. Götz ME, Künig G, Riederer P and Youdim MB: Oxidative stress: free radical production in neural degeneration. Pharmacol Ther 63: 37-122, 1994.
27. Ling YH, Liebes L, Zou Y and Perez-Soler R: Reactive oxygen species generation and mitochondrial dysfunction in the apoptotic response to Bortezomib, a novel proteasome inhibitor, in human $\mathrm{H} 460$ non-small cell lung cancer cells. J Biol Chem 278: 33714-33723, 2003.

28. Cagnol S and Chambard JC: ERK and cell death: mechanisms of ERK-induced cell death-apoptosis, autophagy and senescence. FEBS J 277: 2-21, 2010.

29. Kralova J, Dvorak M, Koc M and Kral V: p38 MAPK plays an essential role in apoptosis induced by photoactivation of a novel ethylene glycol porphyrin derivative. Oncogene 27: 3010-3020, 2008.

30. Liu LJ,Liu LQ, Bo T, et al: Puerarin Suppress Apoptosis of Human Osteoblasts via ERK Signaling Pathway. Int J Endocrinol 2013: 786574, 2013.

31. Zhu G, Wang X, Wu S and Li Q: Involvement of activation of PI3K/Akt pathway in the protective effects of puerarin against MPP+-induced human neuroblastoma SH-SY5Y cell death. Neurochem Int 60: 400-408, 2012.

32. Hien TT, Kim HG, Han EH, Kang KW and Jeong HG: Molecular mechanism of suppression of MDR1 by puerarin from Pueraria lobata via NF-kappaB pathway and cAMP-responsive element transcriptional activity-dependent up-regulation of AMP-activated protein kinase in breast cancer MCF-7/adr cells. Mol Nutr Food Res 54: 918-928, 2010 